\title{
POSITIVE LINEAR OPERATORS AND SUMMABILITY
}

\author{
J. P. KING and J. J. SWETITS
}

(Received 20 June 1968, revised 9 December 1968)

Communicated by J. B. Miller

\section{Introduction}

Let $\left\{L_{n}\right\}$ be a sequence of positive linear operators defined on $C[a, b]$ of the form

$$
L_{n}(f)(x)=\sum_{k=0}^{\infty} a_{n k}(x) f\left(x_{n k}\right)
$$

where $x_{n k} \in[a, b]$ for each $k=0,1, \cdots, n=1,2, \cdots$. The convergence properties of the sequences $\left\{L_{n}(f)\right\}$ to $f$ for each $f \in C[a, b]$ have been the object of much recent research (see e.g. [4], [8], [11], [13]). In many cases positive linear operators of the form (1) give rise to interesting summability matrices $A=\left(a_{n k}(x)\right)$ and viceversa.

It is the object of this paper to give some results which show that if the positive linear operators defined by (1) have the proper convergence properties, then the matrix $A=\left(a_{n k}(x)\right)$ is always a regular summability matrix for certain values of the parameter $x$, and to present certain applications of these results. The theorems and the applications indicate that sequences of positive linear operators provide a rich supply of regular matrices.

Moreover the connection between the almost convergence properties of the operators defined by (1) and the almost regularity of the associated matrix $A=\left(a_{n k}(x)\right)$ are investigated.

Throughout the paper the point of view that is adopted is that a sequence of positive linear operators $\left\{L_{n}\right\}$, defined by equation (1), is given and that this sequence has certain prescribed convergence properties. The matrix $A=\left(a_{n k}\right)$ defined by (1) is considered to be independent of the sequence of points $\left\{x_{n k}\right\}$. A classical example of operators of this type, the Bernstein polynomials, is discussed in section 3 .

The well-known Fejer-Hermite operator $L_{n}$, with nodes at the zeros $x_{i}$ of the $n$th Tchebycheff polynomial $T_{n}[3$, p. 70], defined by

$$
L_{n}(f)(x)=\frac{1}{n^{2}} T_{n}^{2}(x) \sum_{i=1}^{n} f\left(x_{i}\right) \frac{1-x x_{i}}{\left(x-x_{i}\right)^{2}}
$$


provide an example of the kind of operators which are not considered in this paper. (Because if the Fejer-Hermite operator is written in the form of equation (1) the resulting matrix $\left(a_{n k}\right)$ is clearly dependent on the sequence of points $x_{i}$.)

The problem of determining a sequence of points $\left\{x_{n k}\right\}$ corresponding to a given matrix $A=\left(a_{n k}(x)\right)$ such that the resulting operator defined by equation (1) is convergent for each $f$ in a certain class of functions is another matter and no results in this direction are given here. Some results of this type for operators generated by certain classes of analytic functions may be found in the paper of Boehme and Powell [2].

\section{Convergent positive linear operators}

It is clear that if $a_{n k}(x) \geqq 0$ for each $k=0,1, \cdots, n=1,2, \cdots$, and $x \in[a, b]$, then the linear operator defined by $(1)$ is positive on $C[a, b]$. The following theorem is a sort of converse to this result:

THEOREM 1. Let $\left\{L_{n}\right\}$ be a sequence of positive linear operators on $C[a, b]$ defined by (1) for $n=1,2, \cdots$. Let $A=\left(a_{n k}(x)\right)$ be the infinite matrix defined by (1) where, in addition, $a_{00}(x)=1, a_{0 k}(x)=0$ for $k=1,2, \cdots$ and $x \in[a, b]$. Suppose that $a \leqq x_{n k}<x_{n, k+1} \leqq b, k=0,1, \cdots$ and $n=1,2, \cdots$. Then $a_{n k}(x) \geqq 0$ for each $k, n=0,1,2, \cdots$ and $x \in[a, b]$.

Proof. Let $n \geqq 1, k \geqq 0$. Suppose $x_{n k}=a$, i.e. $k=0$. Let $g_{k}^{n} \in C[a, b]$ be defined by

$$
g_{k}^{n}(\xi)=\left\{\begin{array}{cl}
1 & \xi=a \\
1+\frac{\xi-a}{a-x_{n, k+1}} & a<\xi \leqq x_{n, k+1} \\
0 & x_{n, k+1}<\xi \leqq b .
\end{array}\right.
$$

Then $g_{k}^{n}(\xi) \geqq 0$ for $\xi \in[a, b]$ and, since $L_{n}$ is a positive operator, $L_{n}\left(g_{k}^{n}\right)(x) \geqq 0$ for $x \in[a, b]$. But $L_{n}\left(g_{k}^{n}\right)(x)=a_{n k}(x)$. Thus $a_{n k}(x) \geqq 0$ for $x \in\left[a_{;} b\right]$.

It $a<x_{n k}$, let $h_{k}^{n} \in C[a, b]$ be defined by

$$
h_{k}^{n}(\xi)=\left\{\begin{array}{cl}
0 & \xi=a \\
1+\frac{\xi-x_{n k}}{x_{n k}-a} & a<\xi \leqq x_{n k} \\
1+\frac{\xi-x_{n k}}{x_{n k}-x_{n, k+1}} & x_{n k}<\xi \leqq x_{n, k+1} \\
0 & x_{n, k+1}<\xi \leqq b
\end{array}\right.
$$

Therefore $L_{n}\left(h_{k}^{n}\right)(x)=a_{n k}(x) \geqq 0$ for each $x \in[a, b]$.

It is now possible to use Theorem 1 to produce a theorem of the type promised in the introduction. 
THEOREM 2. Let $\left\{L_{n}\right\}$ be a sequence of positive linear operators on $C[a, b]$ defined by (1) for $n=0,1, \cdots$. Let $A=\left(a_{n k}(x)\right)$ be defined by (1) with $a_{00}(x)=1$, $a_{0 k}(x)=0$ for $k=1,2, \cdots$, and $x \in[a, b]$. Let $a \leqq x_{n k}<x_{n, k+1} \leqq b$ for each $k=0,1, \cdots$, and $n=1,2, \cdots$, and $\lim _{n \rightarrow \infty} x_{n k}=a, k=0,1, \cdots$. If $\lim _{n \rightarrow \infty} L_{n}(f)(x)$ $=f(x)$ for each $f \in C[a, b]$ and $x \in[a, b]$, then $A=\left(a_{n k}(x)\right)$ is a regular summability matrix for each $x \in[a, b]$.

Proof. Let $e^{0}(x)=1$ for each $x \in[a, b]$. Then $e^{0} \in C[a, b]$ and hence $\lim _{n \rightarrow \infty} L_{n}\left(e^{0}\right)(x)=1$ for each $x \in[a, b]$. Consequently,

$$
\lim _{n \rightarrow \infty} \sum_{k=0}^{\infty} a_{n k}(x)=1, \quad x \in[a, b] .
$$

It follows from Theorem 1 that $a_{n k}(x) \geqq 0$ for each $x \in[a, b]$. Hence

$$
\sup \left\{\sum_{k=0}^{\infty}\left|a_{n k}(x)\right|: n=0.1, \cdots\right\}<\infty, \quad x \in[a, b] .
$$

Let $x \in(a, b]$ and let $k$ be a fixed non-negative integer. Let $N$ be a positive integer such that $n \geqq N$ implies $a \leqq x_{n, k+1}<x \leqq b$. Let $g_{k}^{N} \in C[a, b]$ be defined by (2). Let $M>N$ be such that $g_{k}^{N}\left(x_{n k}\right)>\frac{1}{2}$ for $n \geqq M$. It follows that

$$
0 \leqq \frac{1}{2} a_{n k}(x) \leqq \sum_{p=0}^{\infty} a_{n p}(x) g_{k}^{N}\left(x_{n p}\right)
$$

for $n \geqq M$. Since $g_{k}^{N} \in C[a, b]$ and $g_{k}^{N}(x)=0$, it follows that

Therefore

$$
\lim _{n \rightarrow \infty} \sum_{p=0}^{\infty} a_{n p}(x) g_{k}^{N}\left(x_{n p}\right)=0 .
$$

$$
\lim _{n \rightarrow \infty} a_{n k}(x)=0 .
$$

It follows from (4), (5), (6), and the Silverman-Toeplitz Theorem that $A=$ $\left(a_{n k}(x)\right)$ is regular for each $x \in(a, b]$.

For certain special classes of linear operators of the form (1) it is possible to remove the assumption of positivity and the condition $\lim _{n \rightarrow \infty} x_{n k}=a$. The following theorem gives a result in this direction.

THEOREM 3. Let $\left\{L_{n}\right\}$ be a sequence of linear operators on $C[a, b]$ of the form

$$
L_{n}(f, x)=\sum_{k=n}^{\infty} a_{n k}(x) f\left(x_{n k}\right), \quad n=0,1, \cdots
$$

where $a \leqq x_{n k}<x_{n, k+1} \leqq b$ for each $n, k=0,1, \cdots$

If $\lim _{n \rightarrow \infty} L_{n}(f, x)=f(x)$ for each $f \in C[a, b]$ and $x \in[a, b]$, then $A=\left(a_{n k}(x)\right)$ is a regular summability matrix for each $x \in[a, b]$. 
Proof. Since $\lim _{n \rightarrow \infty} L_{n}\left(e^{0}, x\right)=1$, it follows that

$$
\lim _{n \rightarrow \infty} \sum_{k=n}^{\infty} a_{n k}(x)=1, \quad x \in[a, b] .
$$

It is clear that

$$
\lim _{n \rightarrow \infty} a_{n k}(x)=0, \quad x \in[a, b], k=0,1, \cdots
$$

since $a_{n k}(x)=0$ for $k<n$.

Fix $x \in[a, b]$. Then $L_{n}(, x]$ is a linear operator from $C[a, b]$ to $R$, where $R$ is the set of real numbers. Let

$$
\|f\|=\sup _{a \leqq t \leqq b}|f(t)| \text { and } \quad L_{n}(, x) \|=\sup _{\|f\| \leqq 1}\left|L_{n}(f, x)\right| .
$$

Let $L_{n}^{m}(, x)$ be defined by

$$
L_{n}^{m}(f, x)=\sum_{k=n}^{m} a_{n k}(x) f\left(x_{n k}\right) .
$$

It is clear that $L_{n}^{m}(, x)$ is a continuous linear operator from $C[a, b]$ to $R$. Since $\lim _{m \rightarrow \infty} L_{n}^{m}(f, x)=L_{n}(f, x)$, the Banach-Steinhaus Closure Theorem [12, p. 117] implies that $L_{n}(, x)$ is a continuous linear operator from $C[a, b]$ to $R$.

Since $\lim _{n \rightarrow \infty} L_{n}(f, x)=f(x),\left|L_{n}(f, x)\right|<1+|f(x)|$ for all but a finite number of $n$. Thus $\left\{L_{n}(, x)\right\}$ is a pointwise bounded sequence of continuous linear operators from $C[a, b]$ to $R$. By the uniform boundedness principle [12, p. 116], $\left\{L_{n}(, x)\right\}$ is uniformly bounded.

Fix $\xi \in[a, b]$ and let $m>n$ where $n$ is a fixed positive integer. Define $f(x)$ as follows:

$$
\begin{aligned}
& f(x)= \\
& \begin{cases}0, & a \leqq x \leqq x_{n, n-1}, \\
\frac{\operatorname{sgn} a_{n n}(\xi)}{x_{n, n}-x_{n, n-1}}\left(x-x_{n, n-1}\right), & x_{n, n-1}<x \leqq x_{n, n}, \\
\frac{\operatorname{sgn} a_{n, n+1}(\xi)-\operatorname{sgn} a_{n n}(\xi)}{x_{n, n+1}-x_{n n}}\left(x-x_{n n}\right)+\operatorname{sgn} a_{n n}(\xi), & x_{n n}<x \leqq x_{n, n+1}, \\
\vdots \quad & \\
\frac{\operatorname{sgn} a_{n m}(\xi)-\operatorname{sgn} a_{n, m-1}(\xi)}{x_{n m}-x_{n, m-1}}\left(x-x_{n, m-1}\right)+\operatorname{sgn} a_{n, m-1}(\xi), & x_{n, m-1}<x \leqq x_{n m}, \\
\frac{\operatorname{sgn} a_{n m}(\xi)}{x_{n m}-x_{n, m+1}}\left(x-x_{n, m+1}\right), & x_{n m}<x \leqq x_{n, m+1}, \\
0, & x_{n, m+1}<x \leqq b .\end{cases}
\end{aligned}
$$

Then $L_{n}(f, \xi)=\sum_{k=n}^{m}\left|a_{n k}(\xi)\right|$. Since $\|f\|=1, \sum_{k=n}^{m}\left|a_{n k}(\xi)\right| \leqq\left\|L_{n}(, \xi)\right\|$. Because $m$ is arbitrary, $\sum_{k=n}^{\infty}\left|a_{n k}(\xi)\right| \leqq\left\|L_{n}(, \xi)\right\|$. Therefore, 


$$
\begin{gathered}
\quad \sup \left\{\sum_{k=n}^{\infty}\left|a_{n k}(\xi)\right|: n=0,1, \cdots\right\} \\
\leqq \sup \left\{\left\|L_{n}(, \xi)\right\|: n=0,1, \cdots\right\}<\infty .
\end{gathered}
$$

The theorem follows from (7), (8), (9), and the Silverman-Toeplitz Theorem.

\section{Applications for convergent positive linear operators}

i) For each $f \in C[0,1]$ let $B_{n}(f)$ be defined by

$$
B_{n}(f)(x)=\sum_{k=0}^{n}\left(\begin{array}{l}
n \\
k
\end{array}\right) x^{k}(1-x)^{n-k} f\left(\frac{k}{n}\right)=\sum_{k=0}^{\infty} a_{n k}(x) f\left(\frac{k}{n}\right)
$$

for each $n=1,2, \cdots B_{n}(f)$ is the $n^{\text {th }}$ Bernstein polynomial and it is well known that $\lim _{n \rightarrow \infty} B_{n}(f)(x)=f(x)$ uniformly on $[0,1]$ for each $f \in C[0,1]$. With $a_{00}(x)$ $=1$ and $a_{0 k}(x)=0$ for $k=1,2, \cdots$ and $x \in[0,1]$, the matrix $A=\left(a_{n k}(x)\right)$ becomes the Euler matrix [1]. Theorem 2 then gives the well known result that the Euler matrix is regular for $0<x \leqq 1$.

A slight modification of the Bernstein polynomials shows that the converse to Theorem 2 is not true. More precisely, let

$$
T_{n}(f)(x)=\sum_{k=0}^{n}\left(\begin{array}{l}
n \\
k
\end{array}\right)\left(x^{2}\right)^{k}\left(1-x^{2}\right)^{n-k} f\left(\frac{k}{n}\right),
$$

where $f \in C[0,1]$ and $x \in[0,1]$. The matrix $A=\left(a_{n k}(x)\right)$ is the Euler matrix. Let $e^{1}(x)=x$. Then it is easy to see that $T_{n}\left(e^{1}\right)(x)=x^{2}$. Hence the regularity of $A$ does not imply that $\lim _{n \rightarrow \infty} T_{n}(f)(x)=f(x)$.

(ii) Let $h_{p}=h_{p}(x)$ be defined on $[0,1]$ for each $p=1,2, \cdots$. Let $A=$ $\left(a_{n k}(x)\right)$ be defined by $a_{00}(x)=1, a_{0 k}(x)=0, k=1,2, \cdots$, and

$$
\prod_{p=1}^{n}\left[z h_{p}(x)+1-h_{p}(x)\right]=\sum_{k=0}^{\infty} a_{n k}(x) z^{k}
$$

Let $\left\{L_{n}\right\}$ be defined by

$$
L_{n}(f)(x)=\sum_{k=0}^{\infty} a_{n k}(x) f\left(\frac{k}{n}\right),
$$

for each $n=1,2, \cdots, x \in[0,1]$ and $f \in C[0,1]$. If $0 \leqq h_{p} \leqq 1, p=1,2, \cdots$, then $L_{n}$ is a positive linear operator on $C[0,1]$ for each $n=1,2, \cdots$. It is shown in [8] that $\lim _{n \rightarrow \infty} L_{n}(f)(x)=f(x)$ uniformly on $[0,1]$ for each $f \in C[0,1]$ if the sequence $\left\{h_{p}(x)\right\}$ is uniformly $(C, 1)$ summable to $x$ on $[0,1]$. It therefore follows from Theorem 2 that, under these conditions, $A=\left(a_{n k}(x)\right)$ is a regular matrix for $x \in(0,1]$. The matrix $A(x)$ is the $\left(F, d_{n}(x)\right)$ or generalized Lototsky matrix [6] where $d_{n}(x)=\left(1-h_{n}(x) / h_{n}(x)\right)$. 
(iii) For each $f \in C[0,1]$ let $P_{n}(f)$ be defined by

$$
P_{n}(f)(x)=(1-x)^{n} \sum_{k=n}^{\infty}\left(\begin{array}{l}
k-1 \\
n-1
\end{array}\right) x^{k-n} f\left(\frac{k-n}{k}\right)
$$

for each $n=1,2, \cdots$. It was shown by Meyer-Konig and Zeller [11] that $\lim _{n \rightarrow \infty}$ $P_{n}(f)(x)=f(x)$ uniformly on $\left[x_{1}, x_{2}\right]$ where $0 \leqq x_{1}<x_{2}<1$ for each $f \in C[0,1]$. It follows from Theorem 3 that the associated matrix $A=\left(a_{n k}(x)\right)$ is a regular summability matrix for each $x \in\left[x_{1}, x_{2}\right]$. In this case the matrix $A=\left(a_{n k}(x)\right)$ is essentially the Taylor matrix [5].

Since the Euler matrix $E(x)$ is regular if and only if $x \in(0,1]$, example (i) shows that Theorem 2 cannot be extended to $[a, b]$.

\section{Almost convergent positive linear operators}

A sequence $x \in m$ is called almost convergent to $s$ if $L(x)=s$ for each Banach limit $L$. The notion of almost convergent sequences was introduced by Lorentz [10].

The following result gives necessary and sufficient conditions on a sequence of positive linear operators $\left\{L_{n}\right\}$ in order that $\left\{L_{n}(f)\right\}$ shall be almost convergent to $f$ for each $f \in C[a, b]$. The proof is identical to the standard proof of the theorem of Korovkin [9, p. 14] for convergent sequences of positive linear operators except for the last few lines. The proof is given here, however, for the sake of completeness.

THEOREM 4. Let $\left\{L_{n}\right\}$ be a sequence of positive linear operators on $C[a, b]$. Let $e^{i} \in C[a, b]$ be defined by $e^{i}(x)=x^{i}$ for $i=0,1,2$. Then the sequence $\left\{L_{n}(f)(x)\right\}$ is almost convergent to $f(x)$ uniformly on $[a, b]$ for each $f \in C[a, b]$ if and only if $\left\{L_{n}\left(e^{i}\right)(x)\right\}$ is almost convergent to $e^{i}(x)$ uniformly on $[a, b]$ for $i=0,1,2$.

Proof. The necessity is obvious. Suppose the conditions on $\left\{L_{n}\left(e^{i}\right)(x)\right\}$ hold for $i=0,1,2$. Let

$$
t_{p v}\left(e^{i}\right)(x)=\frac{1}{p} \sum_{n=v}^{v+p-1} L_{n}\left(e^{i}\right)(x)
$$

for each $v=0,1, \cdots, p=1,2, \cdots$ and $i=0,1,2$. It follows from the definition of almost convergence of $\left\{L_{n}\left(e^{i}\right)(x)\right\}$ to $e^{i}(x)$, a result of Lorentz [10, p. 170], and the hypothesis, that

$$
t_{p v}\left(e^{i}\right)(x)=e^{i}(x)+\alpha_{p v}^{i}(x)
$$

where $\lim _{p \rightarrow \infty} x_{p v}^{i}(x)=0$ uniformly in $v=0,1, \cdots$ and uniformly on $[a, b]$ for each $i=0,1,2$. Let $f \in C[a, b]$ and $\in>0$. Let

$$
t_{p v}(f)(x)=\frac{1}{p} \sum_{n=v}^{v+p-1} L_{n}(f)(x)
$$


for each $p=1,2, \cdots$ and $v=0,1, \cdots$. Choose $\delta>0$ such that $|s-t|<\delta \Rightarrow$ $|f(s)-f(t)|<\varepsilon$. Let $\xi, x \in[a, b]$. Either $|\xi-x| \geqq \delta$ or $|\xi-x|<\delta$.

In the first case it follows that

$$
|f(\xi)-f(x)| \leqq c \varphi(x)
$$

where $c=2\|f\| / \delta^{2}$ and $\varphi(x)=(\xi-x)^{2}$. Therefore

$$
-c \varphi(x)-\varepsilon \leqq f(\xi)-f(x) \leqq c \varphi(x)+\varepsilon .
$$

If $|\xi-x|<\delta$ then $\mid f(\xi)-f(x)<\varepsilon$. Hence the inequality (11) holds in either case. Since $L_{n}$ is linear and positive for each $n=0,1, \cdots$, it follows that

$$
-c L_{n}(\varphi)(x)-\varepsilon L_{n}\left(e^{0}\right)(x) \leqq f(\xi) L_{n}\left(e^{0}\right)(x)-L_{n}(f)(x) \leqq c L_{n}(\varphi)(x)+\varepsilon L_{n}\left(e^{0}\right)(x) .
$$

Therefore

$$
-c t_{p v}(\varphi)(x)-\varepsilon t_{p v}\left(e^{0}\right)(x) \leqq f(\xi) t_{p v}\left(e^{0}\right)(x)-t_{p v}(f)(x)<c t_{p v}(\varphi)(x)+t_{p v}\left(e^{0}\right)(x) .
$$

Hence

$$
\left|f(\xi) t_{p v}\left(e^{0}\right)(\xi)-t_{p v}(f)(\xi)\right| \leqq c t_{p v}(\varphi)(\xi)+\varepsilon t_{p v}\left(e^{0}\right)(\xi)
$$

for each $p=1,2, \cdots, v=0,1, \cdots$. It follows from a simple computation that

$$
t_{p v}(\varphi)(\xi)=\xi^{2} \alpha_{p v}^{1}(\xi)-2 \alpha_{p v}^{2}(\xi)+\alpha_{p v}^{3}(\xi) .
$$

Let $A_{p v}(\xi)=\xi^{2} \alpha_{p v}^{1}(\xi)-2 \alpha_{p v}^{2}(\xi)+\alpha_{p v}^{3}(\xi)$. It follows from (10), (12), and (13) that

$$
\left|f(\xi) t_{p v}\left(e^{0}\right)(\xi)-t_{p v}(f)(\xi)\right| \leqq A_{p v}(\xi)+\varepsilon\left[1+\alpha_{p v}^{1}(\xi)\right]
$$

for each $p=1,2, \cdots$ and $v=0,1, \cdots$. Let $B_{p v}(\xi)=A_{p o}(\xi)+\varepsilon\left[1+\alpha_{p v}^{1}(\xi)\right]$. Then $\lim _{p \rightarrow \infty} B_{p v}(\xi)=\varepsilon$ uniformly in $v=0,1, \cdots$ and uniformly on $[a, b]$. Therefore,

$$
\begin{aligned}
\left|t_{p v}(f)(\xi)-f(\xi)\right| & \leqq\left|t_{p v}(f)(\xi)-f(\xi) t_{p v}\left(e^{0}\right)(\xi)\right|+\left|f(\xi) t_{p v}(f)(\xi)-f(\xi)\right| \\
& \leqq B_{p v}(\xi)+\|f\| \cdot\left|\alpha_{p v}(\xi)\right| .
\end{aligned}
$$

Since $\lim _{p \rightarrow \infty} B_{p v}(\xi)+\|f\| \cdot\left|\alpha_{p v}(\xi)\right|=\varepsilon$ uniformly in $v=0,1, \cdots$ and uniformly on $[a, b]$, the proof is complete.

The proof of the following theorem may be extrapolated from the proof of the trigonometric version of Korovkin's theorem $[9$, p. 17] in a manner similar to that used in the above.

THEOREM 5. Let $\bar{C}[0 ; 2 \pi]$ be the linear space of periodic continuous functions of period $2 \pi$. Let $\left\{L_{n}\right\}$ be a sequence of positive linear operators defined on $\bar{C}[0,2 \pi]$. The sequence $\left\{L_{n}(f)(x)\right\}$ is almost convergent to $f(x)$ uniformly on $[0,2 \pi]$ for each $f \in C[0,2 \pi]$ if and only if $\left\{L_{n}\left(e^{0}\right)(x)\right\},\left\{L_{n}(\cos )(x)\right\},\left\{L_{n}(\sin )(x)\right\}$ are almost convergent to $e^{0}(x), \cos x$, and $\sin x$, respectively, uniformly on $[0,2 \pi]$.

The notion of almost regular matrices was introduced by King [7]. It is pos- 
sible to establish results which relate the almost convergence of a sequence of positive linear operators to the almost regularity of the associated matrix which are similar to those for convergent sequences of positive linear operators, which were given in section 2 . The following theorem is a typical result in this direction:

THEOREM 6. Let $\left\{L_{n}\right\}$ be a sequence of positive linear operators on $C[a, b]$ defined by (1) for $n=1,2, \cdots$. Let $A=\left(a_{n k}(x)\right)$ be the infinite matrix defined by (1) where in addition, $a_{00}(x)=1, a_{0 k}(x)=0$ for $k=1,2, \cdots$ and $x \in[a, b]$. Let $a \leqq x_{n k}<x_{n, k+1} \leqq b$ for each $k=0,1, \cdots$ and $n=1,2, \cdots$, and $\lim _{n \rightarrow \infty} x_{n k}=a$, $k=0,1, \cdots$ If $\left\{L_{n}(f)(x)\right\}$ is almost convergent to $f(x)$ for each $f \in C[a, b]$, and each $x \in[a, b]$, then $A=\left(a_{n k}(x)\right)$ is almost regular for each $x \in(a, b]$.

PRoof. The proof consists of a demonstration that the following conditions, which are necessary and sufficient that $A=\left(a_{n k}(x)\right)$ be almost regular [7], are valid for each $x \in(a, b]$ :

$$
\lim _{p \rightarrow \infty} \frac{1}{p} \sum_{n=v}^{v+p-1} \sum_{k=0}^{\infty} a_{n k}(x)=1
$$

uniformly in $v=0,1, \cdots$

$$
\lim _{p \rightarrow \infty} \frac{1}{p} \sum_{n=v}^{v+p-1} a_{n k}(x)=0, \quad k=0,1, \cdots
$$

uniformly in $v=0,1, \cdots$

and

$$
\sup \left\{\sum_{k=0}^{\infty}\left|a_{n k}(x)\right|: n=0,1, \cdots\right\}<\infty .
$$

This demonstration is entirely analogous to the proof of Theorem 2 and will be omitted.

\section{An application for almost convergent operators}

Let $\left\{h_{i}\right\}$ be a sequence of functions defined on $[0,1]$ with $0 \leqq h_{i}(x) \leqq 1$ for each $i=1,2, \cdots$ and $x \in[0,1]$. Let $\left\{\lambda_{n}(x)\right\}$ be a sequence of functions defined on $[0,1]$ such that $0 \leqq \lambda_{n}(x)$ for each $n=1,2, \cdots$ and $x \in[0,1]$. Let $B=\left(b_{n k}(x)\right)$ be defined by

$$
\begin{gathered}
b_{00}=1, \quad b_{0 k}=0 \quad k>0 \\
\lambda_{n}(x) \prod_{i=1}^{n}\left(h_{i}(x) y+1-h_{i}(x)\right)=\sum_{k=0}^{n} b_{n k}(x) y^{k} .
\end{gathered}
$$

For each $n=1,2, \cdots$, let the operator $L_{n}$ be defined by

$$
L_{n}(f)(x)=\sum_{k=0}^{n} b_{n k}(x) f\left(\frac{k}{n}\right)
$$


for each $f \in C[0,1]$. In the special case $\lambda_{n}(x)=1$ for each $n=1,2, \cdots$ and $x \in[0,1], L_{n}(f)(x)$ reduces to the operator given in section 3 . If, in addition, $h_{i}(x)=x$ for each $i=1,2, \cdots$ and $x \in[0,1], L_{n}(f)$ reduces to the $n^{\text {th }}$ Bernstein polynomial.

THEOREM 7. Let $\left\{L_{n}\right\}$ be the sequence of operators defined in the above. Let $\left\{S_{n}(x)\right\}$ denote the $(C, 1)$ transform of $\left\{h_{i}(x)\right\}$. Suppose that $\left\{S_{n}(x)\right\}$ converges uniformly to $x$ on $[0,1]$ and $\left\{\lambda_{n}(x)\right\}$ is almost convergent to 1 uniformly on $[0,1]$. Then $\left[L_{n}(f)(x)\right\}$ is almost convergent to $f(x)$ uniformly on $[0,1]$ for each $f \in C[0,1]$.

Proof. It is clear that $L_{n}$ is a positive linear operator on $C[0,1]$ for each $n=1,2, \cdots$. Moreover $b_{n k}(x)=\lambda_{n}(x) a_{n k}(x)$ where $A=\left(a_{n k}(x)\right)$ is the matrix studied in [8]. It follows, therefore, from the computations made in [8] that

$$
\begin{aligned}
& L_{n}\left(e^{0}\right)(x)=\lambda_{n}(x) \\
& L_{n}\left(e^{1}\right)(x)=\lambda_{n}(x) S_{n}(x)
\end{aligned}
$$

and

$$
L_{n}\left(e^{2}\right)(x)=\frac{\lambda_{n}(x)}{n}\left\{S_{n}(x)-t_{n}(x)\right\}+S_{n}^{2}(x)
$$

where $t_{n}(x)$ is the $(C, 1)$ transform of the sequence $\left\{h_{i}^{2}(x)\right\}$.

In order to complete the proof the following lemma is needed.

LEMMA 1. Let $\left\{\alpha_{n}\right\}$ be almost convergent to $\alpha$ and let $\left\{\beta_{n}\right\}$ be convergent to $\beta$. Then $\left\{\alpha_{n} \beta_{n}\right\}$ is almost convergent to $\alpha \beta$.

Proof Of THE Lemma. $\alpha_{n} \beta_{n}=\alpha_{n}\left(\beta_{n}-\beta\right)+\beta \alpha_{n}$ for each $n=1,2, \cdots$. The sequence $\left\{\alpha_{n}\left(\beta_{n}-\beta\right)\right\}$ is convergent to $\theta$ since $\alpha_{n}$ is bounded. Hence, $\left\{\alpha_{n} \beta_{n}\right\}$ is almost convergent to $\alpha \beta$. This proves the lemma.

It now follows from Lemma 1 and (15), (16), and (17) that $\left\{L_{n}\left(e^{i}\right)(x)\right\}$ is almost convergent to $e^{i}(x)$ uniformly on $[0,1]$ for $i=0,1,2$. Theorem 7 therefore follows from Theorem 4.

It is easy to see that the sequence $\left\{\left(1-\frac{1}{2} x\right),\left(1+\frac{1}{2} x\right),\left(1-\frac{1}{2} x\right), \cdots\right\}$ satisfies the hypothesis of Theorem 7 for $\left\{\lambda_{n}(x)\right\}$. With this choice of $\left\{\lambda_{n}(x)\right\}$ and with $h_{i}(x)=x$ for each $i=1,2, \cdots$ and $x \in[0,1]$, the functions $L_{n}(f)$ become a kind of modified Bernstein polynomials. Theorem 7 ensures that they are almost convergent to $f(x)$ uniformly on $[0,1]$, even though they may not converge to $f(x)$.

It now follows from Theorem 6 that $B=\left(b_{n k}(x)\right)$ is almost regular for each $x \in(0,1]$.

In the special case in which these operators reduce to the Bernstein polynomials the matrix $B$ reduces to the Euler matrix $E(x)$. It is shown in [7] that $E(x)$ is almost regular if and only if it is regular. Since $E(x)$ is regular if and only if $x \in(0,1]$, this shows that Theorem 6 cannot be extended to $[a, b]$. 


\section{References}

[1] R. P. Agnew, 'Euler Transformations', Amer. J. Math. 66 (1944), 318-338.

[2] T. K. Boehme and R. E. Powell, 'Positive Linear Operators Generated by Analytic Functions', Siam J. Appl. Math., 16 (1968), 510-519.

[3] E. W. Cheney, Introduction to Approximation Theory (McGraw-Hill, 1966).

[4] E. W. Cheney and A. Sharma, 'Bernstein power series', Can. J. Math. 16 (1964), 241 -252.

[5] V. F. Cowling, 'Summability and analytic continuation', Proc. Amer. Math. Soc. 1 (1950), $536-542$

[6] A. Jakimovski, 'A generalization of the Lototsky method of summability', Michigan Math. J. 6 (1959), 277-290.

[7] J. P. King, 'Almost summable sequences', Proc. Amer. Math. Soc. 17 (1966), 1219-1225.

[8] J. P. King, 'The Lototsky transform and Bernstein polynomials', Can. J. Math. 18 (1966), $89-91$.

[9] P. Korovkin, Linear operators and approximation theory (translated from the Russian edition of 1959, Delhi, 1960).

[10] G. G. Lorentz, 'A contribution to the theory of divergent sequences', Acta. Math. 80 (1948), 167-190.

[11] W. Meyer-Konig and K. Zeller, 'Bernsteinsche Potenzreihen', Studia Math. 19 (1960), $89-94$.

[12] A. Wilansky, Functional Analysis (Blaisdel1, 1964).

[13] H. Bohman, 'On approximation of continuous and of analytic functions', Ark. Mat. 2 (1952) $43-56$.

Lehigh University

Lafayette College 